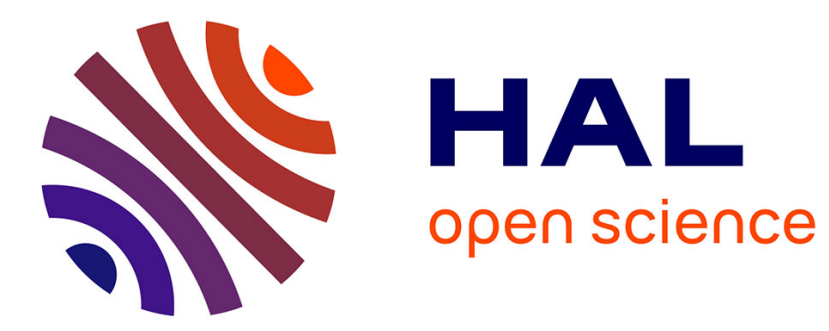

\title{
Non-parametric-parametric model for random uncertainties in non-linear structural dynamics: Application to earthquake engineering
}

\author{
Christophe Desceliers, Christian Soize, S. Cambier
}

\section{- To cite this version:}

Christophe Desceliers, Christian Soize, S. Cambier. Non-parametric-parametric model for random uncertainties in non-linear structural dynamics: Application to earthquake engineering. EARTHQUAKE ENGINEERING \& STRUCTURAL DYNAMICS, 2004, 33 (3), pp.315-327. 10.1002/eqe.352 . hal00686208

\section{HAL Id: hal-00686208 \\ https://hal.science/hal-00686208}

Submitted on 8 Apr 2012

HAL is a multi-disciplinary open access archive for the deposit and dissemination of scientific research documents, whether they are published or not. The documents may come from teaching and research institutions in France or abroad, or from public or private research centers.
L'archive ouverte pluridisciplinaire HAL, est destinée au dépôt et à la diffusion de documents scientifiques de niveau recherche, publiés ou non, émanant des établissements d'enseignement et de recherche français ou étrangers, des laboratoires publics ou privés. 


\title{
Nonparametric-parametric model for random uncertainties in nonlinear structural dynamics - Application to earthquake engineering
}

\author{
Christophe Desceliers ${ }^{1}$, Christian Soize ${ }^{1, *}$, Simon Cambier ${ }^{2}$ \\ ${ }^{1}$ Laboratoire de Mécanique, Université de Marne-La-Vallée, 77454, Marne-La-Vallée Cedex 2, France \\ ${ }^{2}$ EDF R\&D, Analysis in Mechanics and Acoustics Dpt, 92141, Clamart Cedex, France
}

\begin{abstract}
SUMMARY
This paper deals with the transient response of a nonlinear dynamical system with random uncertainties. The nonparametric probabilistic model of random uncertainties recently published and extended to nonlinear dynamical system analysis is used in order to model random uncertainties related to the linear part of the finite element model. The nonlinearities are due to restoring forces whose parameters are uncertain and are modeled by the parametric approach. Jayne's maximum entropy principle with the constraints defined by the available information allows the probabilistic model of such random variables to be constructed. Therefore, a nonparametric-parametric formulation is developped in order to model all the sources of uncertainties in such a nonlinear dynamical system. Finally, a numerical application for earthquake engineering analysis is proposed and concerned a reactor cooling system under seismic loads.
\end{abstract}

KEY WORDS : Nonlinear structural dynamics, Earthquake, Random uncertainties

\section{INTRODUCTION}

This paper deals with the transient response of a nonlinear dynamical system with random uncertainties. The nonlinear dynamical system is constituted of a linear damped elastic structure representing a reactor cooling system. The nonlinearities are due to restoring forces induced by elastic stops modeling supports of the reactor cooling system. For given gaps, these elastic stops limit the vibration amplitudes of the steam generator system. The displacement field of this structure is constrained by several timedependent Dirichlet conditions corresponding to seismic loads operating on the anchors of the reactor cooling system and elastic stops.

* Correspondence to: Christian Soize, Laboratoire de Mécanique, Université de Marne-la-Vallée, 5 bd Descartes, 77454 Marne-laVallée, Cedex 2, France. E-mail : soize@univ-mlv.fr. 
The source of random uncertainties are induced by model uncertainties (model errors) and by the data uncertainties (errors on the parameters of the model). For the problem under consideration, data uncertainties concern the local parameters of the finite element model and the parameters of the nonlinear forces. Usually, parametric approaches are used to model data uncertainties [1-7] for evaluating and analyzing the response of structures with uncertain parameters under seismic loads, like piping and equipment, power plant installations and industrial structures (for instance, see [8-12]). Nevertheless, such approaches do not allow model uncertainties to be taken into account. It should be noted that model uncertainties can only be modeled by using a nonparametric approach. Recently, such a nonparametric model of random uncertainties has been introduced for linear dynamical system $[13,14]$. This nonparametric model has also been extended to the transient response of nonlinear dynamical system [15] without having random uncertainties on the nonlinear part. This nonparametric model is constructed by the use of Jayne's entropy principle which consists in maximizing the entropy with the constraints defined by the available information (for instance, information related to algebraic properties of the finite element matrices). Due to the model uncertainties (for instance, errors induced by the introduction of simplified kinematical assumptions) and due to the data uncertainties related to the local parameters of the finite element model (for instance, geometrical and mechanical parameters), the generalized mass, damping and stiffness matrices of the reduced matrix model corresponding to the linear part of the finite element model are random matrices. The probability distributions of such random matrices are modeled by the probability distribution on the set of all the positive-symmetric real random matrices, introduced in references [13-15]. It should be noted that such a nonparametric formulation allows model uncertainties and data uncertainties of the finite element model to be taken into account. Nevertheless, this nonparametric formulation does not allow the uncertainties related to the nonlinear restoring forces to be modeled. This is the reason why a nonparametric-parametric formulation is proposed in order to take into account model uncertainties and data uncertainties for the linear part of the dynamical system and data uncertainties for the nonlinearities of the dynamical system.

In this paper, the finite element model of the nonlinear dynamical system is called the mean finite element model. Due to time-dependent Dirichlet conditions, the relative displacements with respect to the support are classically introduced to obtain homogeneous Dirichlet conditions. Then, the mean finite element model is rewritten with respect to these relative displacements. External loads are assumed to induce a transient response whose energy is almost entirely distributed over a broad band belonging the low-frequency domain. Consequently, a reduced matrix model can be constructed using the modal analysis with a relatively small number of structural modes. 
The nonparametric-parametric probabilistic model of random uncertainties is then constructed (1) by replacing the generalized mass, damping and stiffness matrices of the linear part of the mean reduced matrix model by full random matrices and (2) by replacing the parameters of the nonlinear restoring forces by real random variables. The probability distributions of these generalized random matrices are constructed in references [13-15]. The probability distributions of the random parameters of the nonlinear restoring forces are constructed using Jayne's entropy principle with the constraints defined by the available information. It should be noted that the external loads are deterministic, but the matrices and the parameters of the reduced matrix model are random variables. Therefore, the transient response is a vector-valued stochastic process and the normalized response spectra are stochastic processes indexed by the frequency band of analysis. Moreover, the transient response and the normalized response spectra are nonlinear functions of the random matrices and of the random parameters. Consequently, the most efficient method for estimating the probabilistic solution of the random nonlinear differential matrix equation is the Monte-Carlo numerical simulation method [16,17].

In Section 2, the mean reduced matrix model of the nonlinear dynamical system is presented. Section 3 is devoted to the construction of the nonparametric-parametric model of uncertainties for the nonlinear dynamical system. In Section 4, the application to the earthquake engineering analysis of a reactor cooling system is presented.

\section{MEAN REDUCED MATRIX MODEL OF THE NONLINEAR DYNAMICAL SYSTEM SUB- JECTED TO SEISMIC LOADS}

We consider a nonlinear dynamical system constituted of a damped structure subjected to $m_{s}$ deterministic time-dependent dirichlet conditions corresponding to seismic loads applied to the supports of the structure. The nonlinearities are due to stops with high stiffness, installed with a given gap at given points of the structure. Let $z$ be the $\mathbb{R}^{m_{f}}$-vector of the $m_{f}$ degrees of freedom corresponding to the total displacements, including the displacements of the supports. We are interested in the transient response $z(t)$ on the time interval $[0, T]$. Let $[\underline{\mathcal{M}}],[\underline{\mathcal{D}}]$ and $[\underline{\mathcal{K}}]$ be the mass, damping and stiffness matrices of the linear part of the mean finite element model. It is assumed that the structure with fixed supports has no rigid body modes. Consequently $[\underline{\mathcal{M}}],[\underline{\mathcal{D}}]$ and $[\underline{\mathcal{K}}]$ are positive-definite symmetric $\left(m_{f} \times m_{f}\right)$ real matrices. Vector $\boldsymbol{z}(t)$ is rewritten as $\boldsymbol{z}(t)=\left(\underline{\mathbf{z}}(t), \underline{\mathbf{z}}_{s}(t)\right)$ in which $\underline{\mathbf{z}}_{s}(t) \in \mathbb{R}^{m_{s}}$ is the vector of the $m_{s}$ constrained DOFs at the supports and where $\underline{\mathbf{z}}(t) \in \mathbb{R}^{m}$ is the vector of the $m$ unconstrained DOFs with 
$m=m_{f}-m_{s}$. The block splitting of matrices $[\underline{\mathcal{M}}],[\underline{\mathcal{D}}]$ and $[\underline{\mathcal{K}}]$ relative to $\boldsymbol{z}(t)=\left(\underline{\mathbf{z}}(t), \underline{\mathbf{z}}_{s}(t)\right)$ are introduced,

$$
[\underline{\mathcal{M}}]=\left[\begin{array}{cc}
{[\underline{\underline{M}}]} & {\left[\underline{\mathbb{M}}_{c}\right]} \\
{\left[\underline{\underline{M}}_{c}\right]^{T}} & {\left[\underline{\underline{M}}_{s}\right]}
\end{array}\right] \quad, \quad[\underline{\mathcal{D}}]=\left[\begin{array}{cc}
{[\underline{\mathbb{D}}]} & {\left[\underline{\mathbb{D}}_{c}\right]} \\
{\left[\underline{\underline{D}}_{c}\right]^{T}} & {\left[\underline{\underline{D}}_{s}\right]}
\end{array}\right] \quad, \quad[\underline{\mathcal{K}}]=\left[\begin{array}{cc}
{\left[\underline{\mathbb{K}}^{\prime}\right]} & {\left[\underline{\mathbb{K}}_{c}\right]} \\
{\left[\underline{\mathbb{K}}_{c}\right]^{T}} & {\left[\underline{\mathbb{K}}_{s}\right]}
\end{array}\right] .
$$

Since $[\underline{\mathcal{M}}],[\underline{\mathcal{D}}]$ and $[\underline{\mathcal{K}}]$ are positive-definite matrices, then $[\underline{\underline{M}}],[\underline{\mathbb{D}}]$ and $[\underline{\mathbb{K}}]$ are also positive-definite symmetric $(m \times m)$ real matrices. Consequently, the inverse matrix $[\underline{\mathbb{K}}]^{-1}$ exists and the matrix $[\underline{R}]=-[\underline{\mathbb{K}}]^{-1}\left[\underline{\mathbb{K}}_{c}\right]$ can be defined. Then, the vector $\underline{\mathbf{y}}(t)=\underline{\mathbf{z}}(t)-[\underline{R}] \underline{\mathbf{z}}_{s}(t)$ of the $m$ relative unconstrained DOFs can be introduced. Let $\omega_{1} \leq \ldots \leq \omega_{n}$ be the eigenfrequencies and let $\varphi_{1}, \ldots, \varphi_{n}$ be the corresponding structural modes such that $[\underline{\mathbb{K}}] \boldsymbol{\varphi}_{\alpha}=\omega_{\alpha}^{2}[\underline{\mathbb{M}}] \boldsymbol{\varphi}_{\alpha}$. Let $\underline{\mathbf{y}}^{n}(t)$ be the projection of $\underline{\mathbf{y}}(t)$ on the subspace of $\mathbb{R}^{m}$ spanned by $\varphi_{1}, \ldots, \varphi_{n}$ with $n \ll m$. We then have $\underline{\mathbf{y}}^{n}(t)=\left[\underline{\Phi}_{n}\right] \underline{\mathbf{q}}^{n}(t)$ in which $\left[\underline{\Phi}_{n}\right]$ is the $(m \times n)$ real matrix whose columns are $\varphi_{1}, \ldots, \varphi_{n}$ and where $\underline{\mathbf{q}}^{n}(t)$ is the vector of the $n$ generalized coordinates. Let the positive-definite symmetric $(n \times n)$ real matrices $\left[\underline{M}_{n}\right],\left[\underline{D}_{n}\right]$ and $\left[\underline{K}_{n}\right]$ be the generalized mass, damping and stiffness matrices of the dynamical system such that

$$
\left[\underline{M}_{n}\right]=\left[\underline{\Phi}_{n}\right]^{T}[\underline{\mathbb{M}}]\left[\underline{\Phi}_{n}\right] \quad, \quad\left[\underline{D}_{n}\right]=\left[\underline{\Phi}_{n}\right]^{T}[\underline{\mathbb{D}}]\left[\underline{\Phi}_{n}\right] \quad, \quad\left[\underline{K}_{n}\right]=\left[\underline{\Phi}_{n}\right]^{T}[\underline{\mathbb{K}}]\left[\underline{\Phi}_{n}\right] \quad .
$$

It can be shown that $\underline{\mathbf{q}}^{n}(t)$ is a solution of the reduced matrix model of the nonlinear dynamical system,

$$
\left[\underline{M}_{n}\right] \underline{\ddot{\mathbf{q}}}^{n}(t)+\left[\underline{D}_{n}\right] \underline{\dot{\mathbf{q}}}^{n}(t)+\left[\underline{K}_{n}\right] \underline{\mathbf{q}}^{n}(t)+\mathbf{F}_{c}^{n}\left(t, \underline{\mathbf{q}}^{n}(t), \underline{\dot{\mathbf{q}}}^{n}(t) ; \underline{\mathbf{w}}\right)=\underline{\mathbf{F}}^{n}(t) \quad, \quad t \in[0, T] \quad,
$$

with the initial conditions,

$$
\underline{\mathbf{q}}^{n}(0)=\underline{\mathbf{q}}^{n}(0)=0
$$

in which $\underline{\mathbf{F}}^{n}(t) \in \mathbb{R}^{m}$ and $\mathbf{F}_{c}^{n}(t, \mathbf{q}, \mathbf{p} ; \underline{\mathbf{w}}) \in \mathbb{R}^{m}$ are such that

$$
\begin{aligned}
& \underline{\mathbf{F}}^{n}(t)=-\left[\underline{\Phi}_{n}\right]^{T}\left([\underline{\mathbb{M}}][\underline{R}]+\left[\underline{\mathbb{M}}_{c}\right]\right) \underline{\underline{\mathbf{z}}}_{s}(t)-\left[\underline{\Phi}_{n}\right]^{T}\left([\underline{\mathbb{D}}][\underline{R}]+\left[\underline{\mathbb{D}}_{c}\right]\right) \underline{\mathbf{z}}_{s}(t), \\
& \mathbf{F}_{c}^{n}(t, \mathbf{p}, \mathbf{q} ; \underline{\mathbf{w}})=\left[\underline{\Phi}_{n}\right]^{T} \mathbb{F}_{c}\left(t,\left[\underline{\Phi}_{n}\right] \mathbf{p}+[\underline{R}] \underline{\mathbf{z}}_{s}(t),\left[\underline{\Phi}_{n}\right] \mathbf{q}+[\underline{R}] \underline{\mathbf{z}}_{s}(t) ; \underline{\mathbf{w}}\right),
\end{aligned}
$$

where $\mathbb{T}_{c}(t, \underline{\mathbf{z}}(t), \underline{\mathbf{z}}(t) ; \underline{\mathbf{w}})$ is the nonlinear forces induced by the stops whose parameters are represented by vector $\underline{\mathbf{w}}=\left(\underline{w}_{1}, \ldots, \underline{w}_{\nu}\right) \in \mathbb{R}^{\nu}$. Hereinafter, it is assumed that the nonlinear dynamical problem defined by Eqs. (3) and (4) has an unique solution $\underline{\mathbf{q}}^{n}(t)$ on $[0, T]$.

\section{STOCHASTIC NONLINEAR DYNAMICAL SYSTEM SUBJECTED TO SEISMIC LOADS}


The nonparametric approach consists in substituting the mean generalized mass, damping and stiffness matrices $\left[\underline{M}_{n}\right],\left[\underline{D}_{n}\right]$ and $\left[\underline{K}_{n}\right]$ of the mean reduced matrix model by the full random matrices $\left[\mathbf{M}_{n}\right]$, $\left[\mathbf{D}_{n}\right]$ and $\left[\mathbf{K}_{n}\right]$. For the problem under consideration, the parametric approach consists in substituting parameter mean values $\underline{\mathbf{w}}$ of the nonlinear forces by the $\mathbb{R}^{\nu}$-valued random variable $\mathbf{W}$. Consequently, the $m$ unconstrained DOFs and the $m$ unconstrained relative DOFs are represented by the $\mathbb{R}^{m}$-valued stochastic processes $\mathbf{Z}^{n}(t)$ and $\mathbf{Y}^{n}(t)$ indexed by $[0, T]$, such that

$$
\begin{gathered}
\mathbf{Z}^{n}(t)=\mathbf{Y}^{n}(t)+[\underline{R}] \underline{\mathbf{z}}_{s}(t) \\
\mathbf{Y}^{n}(t)=\left[\underline{\Phi}_{n}\right] \mathbf{Q}^{n}(t)
\end{gathered}
$$

where the $\mathbb{R}^{n}$-valued stochastic process $\mathbf{Q}^{n}(t)$ indexed by $[0, T]$ is the unique second-order solution of the following stochastic nonlinear differential equation,

$$
\left[\mathbf{M}_{n}\right] \ddot{\mathbf{Q}}^{n}(t)+\left[\mathbf{D}_{n}\right] \dot{\mathbf{Q}}^{n}(t)+\left[\mathbf{K}_{n}\right] \mathbf{Q}^{n}(t)+\mathbf{F}_{c}^{n}\left(t, \mathbf{Q}^{n}(t), \dot{\mathbf{Q}}^{n}(t) ; \mathbf{W}\right)=\underline{\mathbf{F}}^{n}(t) \quad, \quad t \in[0, T],
$$

with the initial conditions,

$$
\mathbf{Q}^{n}(0)=\dot{\mathbf{Q}}^{n}(0)=0
$$

Random matrices $\left[\mathbf{M}_{n}\right],\left[\mathbf{D}_{n}\right]$ and $\left[\mathbf{K}_{n}\right]$ and random vector $\mathbf{W}=\left(W_{1}, \ldots, W_{\nu}\right)$ are second-order random variables subjected to the following constraints defined by the available information,

$$
\begin{gathered}
{\left[\mathbf{M}_{n}\right],\left[\mathbf{D}_{n}\right],\left[\mathbf{K}_{n}\right] \in \operatorname{Mat}_{\mathbb{R}}^{+}(n) \quad \text { a.s }} \\
E\left\{\left[\mathbf{M}_{n}\right]\right\}=\left[\underline{M}_{n}\right], \quad E\left\{\left[\mathbf{D}_{n}\right]\right\}=\left[\underline{D}_{n}\right], \quad E\left\{\left[\mathbf{K}_{n}\right]\right\}=\left[\underline{K}_{n}\right], \\
E\left\{\left\|\left[\mathbf{M}_{n}\right]^{-1}\right\|_{F}^{2}\right\}<+\infty, \quad E\left\{\left\|\left[\mathbf{D}_{n}\right]^{-1}\right\|_{F}^{2}\right\}<+\infty, \quad E\left\{\left\|\left[\mathbf{K}_{n}\right]^{-1}\right\|_{F}^{2}\right\}<+\infty, \\
W_{1} \in \mathcal{D}_{1}, \ldots, W_{\nu} \in \mathcal{D}_{\nu} \quad, \quad \text { a.s }, \\
E\left\{W_{1}\right\}=\underline{w}_{1}, \ldots, E\left\{W_{\nu}\right\}=\underline{w}_{\nu},
\end{gathered}
$$

in which $E$ denotes the mathematial expectation and where $\|[A]\|_{F}=\left(\operatorname{tr}\left\{[A][A]^{T}\right\}\right)^{1 / 2}$. In Eq. (11), $\operatorname{Mat}_{\mathbb{R}}^{+}(n)$ is the set of all the positive-definite symmetric $(n \times n)$ real matrices and in Eq. $(14), \mathcal{D}_{1}, \ldots, \mathcal{D}_{\nu}$ are subsets of $\mathbb{R}$. By construction, $W_{1}, \ldots, W_{\nu}$ are assumed to be $\nu$ indenpendant random variables. Let $Z_{1}(t), \ldots, Z_{n}(t)$ be the coordinates of $\mathbf{Z}^{n}(t)$. Let $S_{j}(\xi, \omega)$ be the random normalized response spectrum of stochastic transient response $Z_{j}^{n}(t)$ with $(\xi, \omega)$ belonging to $J_{\xi} \times J_{\omega} \subset \mathbb{R}^{2}$ where $J_{\xi}=[0,1]$ 
and $J_{\omega}=\left[\omega_{\min }, \omega_{\max }\right]$. We then have

$$
S_{j}(\xi, \omega)=\frac{\omega^{2}}{g} \max _{t \in[0, T]}\left|X_{j}(t)\right|
$$

in which $g$ is a normalization constant (for instance, the gravity acceleration) and where $X_{j}(t)$ is the solution of the linear dynamical system,

$$
\begin{gathered}
\ddot{X}_{j}(t)+2 \xi \omega \dot{X}_{j}(t)+\omega^{2} X_{j}(t)=-Z_{j}^{n}(t) \quad, \quad t \in[0, T] \\
X_{j}(0)=\dot{X}_{j}(0)=0 .
\end{gathered}
$$

Normalized response spectrum $S_{j}(\xi, \omega)$ is a random variable whose mean values $m_{1 j}(\xi, \omega)$, second-order moment $m_{2 j}(\xi, \omega)$, variance $V_{j}(\xi, \omega)$ and standard deviation $\sigma_{j}(\xi, \omega)$ are estimated by the Monte-Carlo numerical simulation. The stochastic convergence of the probabilistic model is studied with respect to $n$ and with respect to the number $n_{s}$ of Monte-Carlo realization, by introducing the norm ||$\left|\ddot{Z}_{j}^{n}\right|||$ defined as

$$
\left\|\ddot{Z}_{j}^{n} \mid\right\|^{2}=E\left\{\int_{0}^{T} \ddot{Z}_{j}^{n}(t)^{2} d t\right\} .
$$

This norm is estimated by ||$\left|\ddot{Z}_{j}^{n}\right| \| \simeq \operatorname{Conv}_{j}\left(n_{s}, n\right)$ with

$$
\operatorname{Conv}_{j}\left(n_{s}, n\right)^{2}=\frac{1}{n_{s}} \sum_{k=1}^{n_{s}} \int_{0}^{T} \ddot{Z}_{j}^{n}\left(t, \theta_{k}\right)^{2} d t,
$$

where $\theta_{1}, \ldots, \theta_{n_{s}}$ denotes the $n_{s}$ realizations for the Monte-Carlo numerical simulation.

Let $d B_{j}(\xi, \omega)$ be the random variable such that $d B_{j}(\xi, \omega)=\log _{10}\left(S_{j}(\xi, \omega)\right)$. The confidence region for the normalized response spectrum $S_{j}(\xi, \omega)$ in $\mathrm{dB}$ associated with the probability level $P_{c}$ is delimited by the upper envelope $d B_{j}^{+}(\xi, \omega)$ and the lower envelope $d B_{j}^{-}(\xi, \omega)$ constructed by using the Chebychev inequality and which are such that

$$
\begin{gathered}
\operatorname{Proba}\left\{d B_{j}^{-}(\xi, \omega)<d B_{j}(\xi, \omega) \leq d B_{j}^{+}(\xi, \omega)\right\} \geq P_{c} \\
d B_{j}^{-}(\xi, \omega)=2 d B_{j}^{0}(\xi, \omega)-d B_{j}^{+}(\xi, \omega) \\
d B_{j}^{+}(\xi, \omega)=\log _{10}\left(m_{1 j}(\xi, \omega)+a_{j}(\xi, \omega)\right) \\
d B_{j}^{0}(\xi, \omega)=\log _{10}\left(m_{1 j}(\xi, \omega)\right) \\
a_{j}(\xi, \omega)=\sigma_{j}(\xi, \omega) /\left(\sqrt{1-P_{c}}\right)
\end{gathered}
$$

The probability model of random matrices $\left[\mathbf{M}_{n}\right],\left[\mathbf{D}_{n}\right]$ and $\left[\mathbf{K}_{n}\right]$ are defined in references [13-15]. The probability model of random variable $\mathbf{W}$ is construct by using Jayne's maximum entropy principle with 
the constraints defined by Eqs. (14) and (15). If $\mathcal{D}_{\ell}=\left[a_{\ell}, b_{\ell}\right]$, then it can be shown that the probability density function $p_{W_{\ell}}(w)$ of random variable $W_{\ell}$ is written as

$$
p_{W_{\ell}}(w)=\mathbb{1}_{\left[a_{\ell}, b_{\ell}\right]}(w) \frac{k_{\ell}}{\alpha_{\ell}\left(k_{\ell}\right)} e^{-k_{\ell} w}
$$

in which $\mathbb{1}_{B}(\omega)$ is is the indicatrix function of the set $\mathrm{B}$ and where the positive real number $k_{\ell}$ is such that $\left(\underline{w}_{\ell} k_{\ell}-1\right) \alpha_{\ell}\left(k_{\ell}\right)-k_{\ell} \beta_{\ell}\left(k_{\ell}\right)=0$ in which $\alpha_{\ell}(k)=e^{-a_{\ell} k}-e^{-b_{\ell} k}$ and $\beta_{\ell}(k)=a_{\ell} e^{-a_{\ell} k}-b_{\ell} e^{-b_{\ell} k}$. If $\mathcal{D}_{\ell}=\left[a_{\ell},+\infty[\right.$, then it can be shown that

$$
p_{W_{\ell}}(w)=\mathbb{1}_{\left[a_{\ell},+\infty[\right.}(w) \frac{e^{-\left(w-a_{\ell}\right) /\left(\underline{w}_{\ell}-a_{\ell}\right)}}{\underline{w}_{\ell}-a_{\ell}} .
$$

If the additional constraint $E\left\{\left(W_{\ell}-a_{\ell}\right)^{-2}\right\}<+\infty$ is introduced, then the probability density function $p_{W_{\ell}}(w)$ is written as

$$
p_{W_{\ell}}(w)=\mathbb{1}_{\left[a_{\ell},+\infty[\right.}(w) \times C_{W_{\ell}} \times\left(w-a_{\ell}\right)^{\left(1-\delta_{\ell}^{2}\right) /\left(\delta_{\ell}^{2}\right)} \times e^{-\left(w-a_{\ell}\right) /\left(\left(\underline{w}_{\ell}-a_{\ell}\right) \delta_{\ell}^{2}\right)},
$$

in which

$$
C_{W_{\ell}}=\left(\underline{w}_{\ell} \delta_{\ell}^{2}-a_{\ell} \delta_{\ell}^{2}\right)^{-\frac{1}{\delta_{\ell}^{2}}} / \Gamma\left(\frac{1}{\delta_{\ell}^{2}}\right)
$$

where the real parameter $\delta_{\ell}>0$ allows the dispersion of random variable $W_{\ell}$ to be fixed. Let $\sigma_{\ell}$ be the standard deviation of random variable $W_{\ell}$. It can be shown that $\delta_{\ell}=\sigma_{\ell} /\left|\underline{w}_{\ell}-a_{\ell}\right|$.

\section{MULTISUPPORTED REACTOR COOLANT SYSTEM SUBJECTED TO SEISMIC LOADS}

The structure under consideration is a typical four loops reactor coolant system [18] (see Fig. 1 on the right). Each loop is constituted of a reactor, a reactor coolant pump and a steam generator (see Fig. 1 on the left). These three elements are connected to each other by three primary coolant pipes: a hot leg which links the reactor with the steam generator, a cold leg which links the reactor with a reactor coolant pump and an intermediate leg which links the reactor coolant with and the steam generator. The curvilinear finite element meshes of a steam generator, of one loop and of the four loops reactor coolant system are shown in Fig. 2. The structure is multisupported with 36 supports (4 times 9 nodes defined as node 1 in Fig. 2 on the middle). Its supports consist in anchors located under the reactor coolant pumps, the steam generators and the cold legs. Due to seismic loads, the displacements of all the 36 supports are constrained by time-dependent Dirichlet conditions. Figure 3 displays an example of accelerogram applied to one of the 36 supports. 
The vibrations of each steam generator are limited by three elastic stops located at their connection point with the intermediate leg and the hot leg (nodes defined as node 3 in Fig. 2 on the left) and located at the middle of each steam generator (nodes defined as node 2 in Fig. 2 on the left). These elastic stops induce nonlinear restoring forces. In addition, the seven elastic stops are subjected to seismic load and consequently, at each stop, the displacements are constrained by time-dependent Dirichlet conditions. The mean finite element model of the four loops reactor coolant system is composed of beam finite elements. Let $j_{\text {stop }}$ be the DOF number corresponding to the horizontal translation of the node $B$ in the steam generator (see Fig. 2). Let $j_{\text {obs }}$ be the DOF number corresponding to the horizontal translation of the node $A$ in the hot leg (see Fig. 2). The first eigeinfrequency of the linear part is $1,4 \mathrm{~Hz}$ and the eigeinfrequency of rank 200 is $164 \mathrm{~Hz}$.

Figure 4 and 5 display the normalized response spectra of the mean nonlinear dynamical system for DOF number $j_{\text {stop }}$ (Fig 4) and for DOF number $j_{\text {obs }}$ (Fig. 5).

The dispersions of random matrices $\left[\mathbf{M}_{n}\right],\left[\mathbf{D}_{n}\right]$ or $\left[\mathbf{K}_{n}\right]$ are controlled by the parameters $\delta_{M}, \delta_{D}$ and $\delta_{K}$ (see reference [14]). Data uncertainties concern the stiffnesses of the stops whose probabilistic models are defined by Eqs. (28) and (29). Random parameters $W_{1}, \ldots, W_{28}$ correspond to the 28 stiffnesses of the 28 elastic stops. Therefore, each parameter belongs to $\mathcal{D}_{\ell}=[0,+\infty[$. Since the structure is multisupported and since the number of nonlinear elastic stops is large, then the solution is very sensitive to the value of the time-step size of the time integration scheme. For the computation, the time-step size $\Delta t$ is chosen as a constant independent of $n$. For each realizations $\theta_{k}$, Eq. (9) is solved by using the Euler explicit step-by-step integration scheme with $\Delta t=1 / 25000 \mathrm{~s}$ and for a time duration $T=15 s$. The Monte-Carlo numerical simulation is performed with $n_{s}=700$ realizations by using a finite element code [19] in which the method presented in this paper has been implemented. Figure 6 is related to the convergence analysis with respect to parameters $n$ and $n_{s}$ and displays the graphs of functions $n_{s} \mapsto \log _{10}\left\{\operatorname{Conv}_{j_{o b s}}\left(n_{s}, n\right)\right\}$ for $n=\{5,50,100,200\}$ and for $\delta_{M}=\delta_{D}=\delta_{K}=0.2$ and $\delta_{1}=\ldots=\delta_{28}=0.2$. It can be seen that convergence with respect to $n$ and $n_{s}$ is obtained for $n=100$ and $n_{s}=280$.

Figures 7 to 10 show the confidence regions for the normalized response spectra in $\mathrm{dB}$, associated with the probability level $P_{c}=0.95$ for $n_{s}=700, n=200, \xi=0.001$. Figures 7 and 9 are relative to DOF $j_{\text {stop }}$ and Figs. 8 and 10 are relative to DOF $j_{\text {obs }}$. Figures 7 and 8 correspond to a nonparametric and a parametric level of uncertainties equal to 0.2 while Figs. 9 and 10 correspond to a nonparametric level of uncertainties equal to 0.00002 and a parametric level of uncertainties equal to 0 . 


\section{COMMENTS AND CONCLUSIONS}

A nonparametric-parametric probabilistic model of random uncertainties has been developed for nonlinear dynamical system in the time domain. A nonparametric probabilistic model of random uncertainties is used for modeling model uncertainties and data uncertainties concerning the linear part of the system. The data uncertainties of the nonlinear part is modeled by the use of a parametric approach.

From the analysis of the confidence region of the normalized response spectra in $\mathrm{dB}$, it can be seen that the responses are very sensitive to uncertainties. For almost zero level of uncertainties, the random responses look like to chaotic responses due to a large number of shocks along the structure. Consequently, it is necessary to model the uncertainties for such a nonlinear dynamical system in order to allow an efficient engineering design process of such a structure to be performed. For such a nonlinear dynamical system, the nonparametric-parametric approach is well adapted.

\section{REFERENCES}

1. Soong TT. Random Differential Equations in Science and Engineering.; Academic Press : New York, 1973.

2. Haug EJ, Choi KK, Komkov V. Design Sensitivity Analysis of Structural Systems; Academic Press : San Diego/London, 1986.

3. Ibrahim RA. Structural dynamics with parameters uncertainties.Applied Mechanics Reviews 1987; 40(3): 309328.

4. Spanos PD, Zeldin BA. Galerkin sampling method for stochastic mechanic problems. Journal of Engineering Mechanics 1994; 120(5): 1091-1106.

5. Iwan D, Jensen $\mathrm{H}$. On the dynamical response of continuous systems including model uncertainty. Transactions of ASME 1993; 60: 484-490.

6. Ghanem R, Spanos PD. Stochastic Finite Elements: A Spectral Approach; Springer-Verlag : New York, 1991 .

7. Ghanem R. Ingredients for a general purpose stochastic finite element formulation. Computer Methodes in Apllied Mechanics and Engineering 1999; 168: 19-34.

8. Zhao YG, Ono T, Idota H. Response uncertainty and time-variant reliability analysis for hysteretic MDF structures. Earthquake Engineering and Structural Dynamics 1999; 28: 1187-1213.

9. de la Llera JC, Chopra AK. Accidental torsion in buiuldings due to stiffness uncertainty. Earthquake Engineering and Structural Dynamics 1994; 23(2): 117-136.

10. Shenton HW, Holloway ES. Effect of stiffness variability on the response of isolated structures. Earthquake Engineering and Structural Dynamics 2000; 29: 19-36.

11. Labbé P, Noe H. Stochastic approach for the seismic design of nuclear power plant equipments. Nuclear Engineering and Design 1991; 180: 367-379.

12. Cambier S, Guihot P, Coffignal G. Computational methods for accounting of structural uncertainties, applications to dynamic behaviour prediction of piping systems. Structural safety 2002; 24: 29-50.

13. Soize C.A nonparametric model of random uncertainties for reduced matrix models in structural dynamics. Probabilistic Engineering Mechanics 2000; 15(3): 277-294.

14. Soize C. Maximum entropy approach for modeling random uncertainties in transient elastodynamics. J. Acoust. Soc. Am. 2001; 109(5): 1979-1996.

15. Soize C. Nonlinear dynamical systems with nonparametric model of random uncertainties.Uncertainties in Engineering Mechanics 2001; http://www.resonance-pub.com; 1(1): 1-38.

16. Rubinstein RY. Simulations and the Monte Carlo Methods; John Wiley and Sons : New York, 1981.

17. Kalos MH, Whitlock PA. Monte Carlo Methods, Volume 1: Basics.; John Wiley and Sons : Chichester, 1986. 
18. Duval C, Guyonvarh V, Louche V, Pigat J, Waeckel F. Estimation methods for seismic behavior of a PWR primary system. 5th National colloque AFPS 1999; 19-21.

19. Code_Aster, Numerical simulation software for structural analysis, Free software developed by EDF under GNU General Public License, www.code-aster.org. 

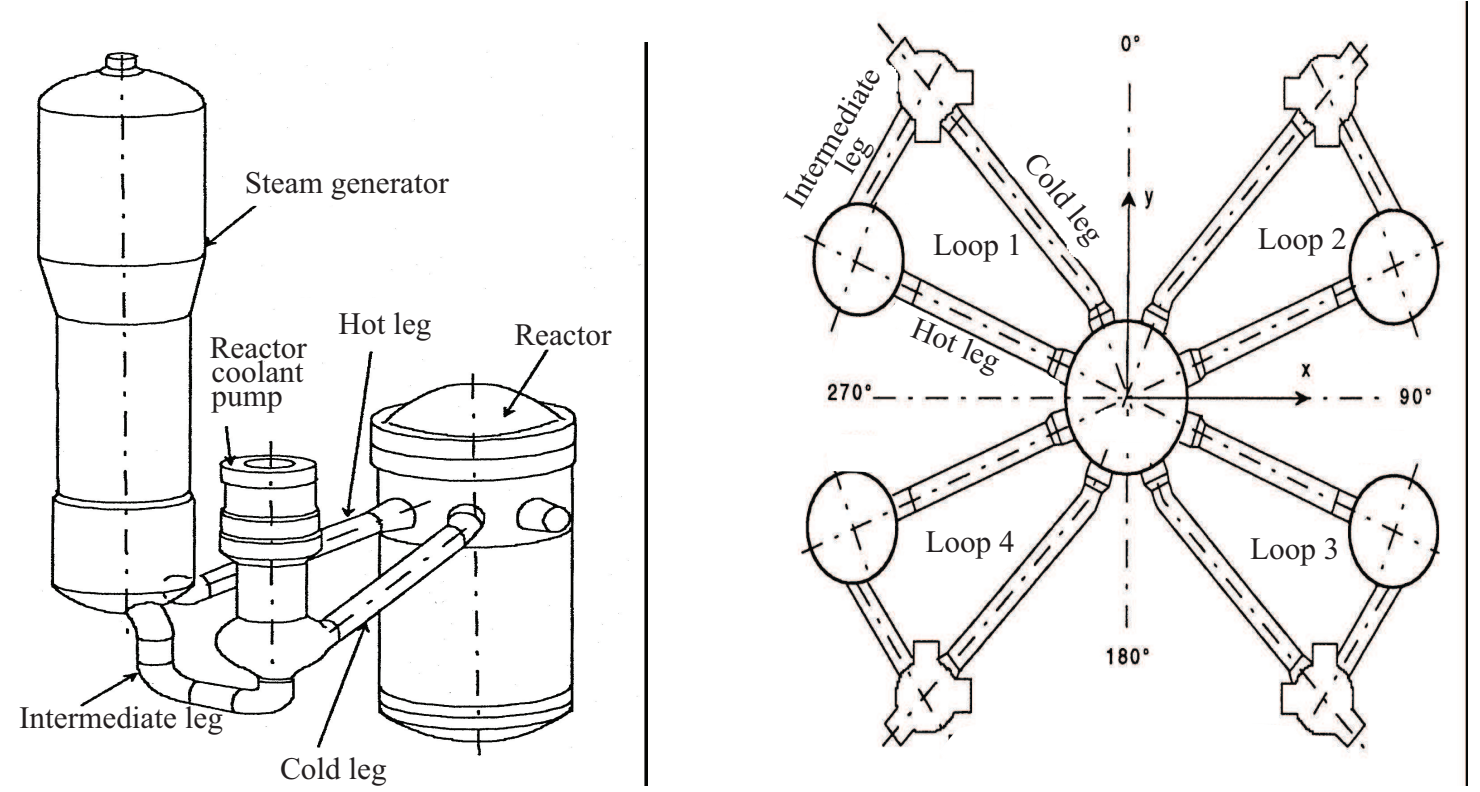

Figure 1. Four loops reactor coolant system (figure on the right). One loop (figure on the left). 


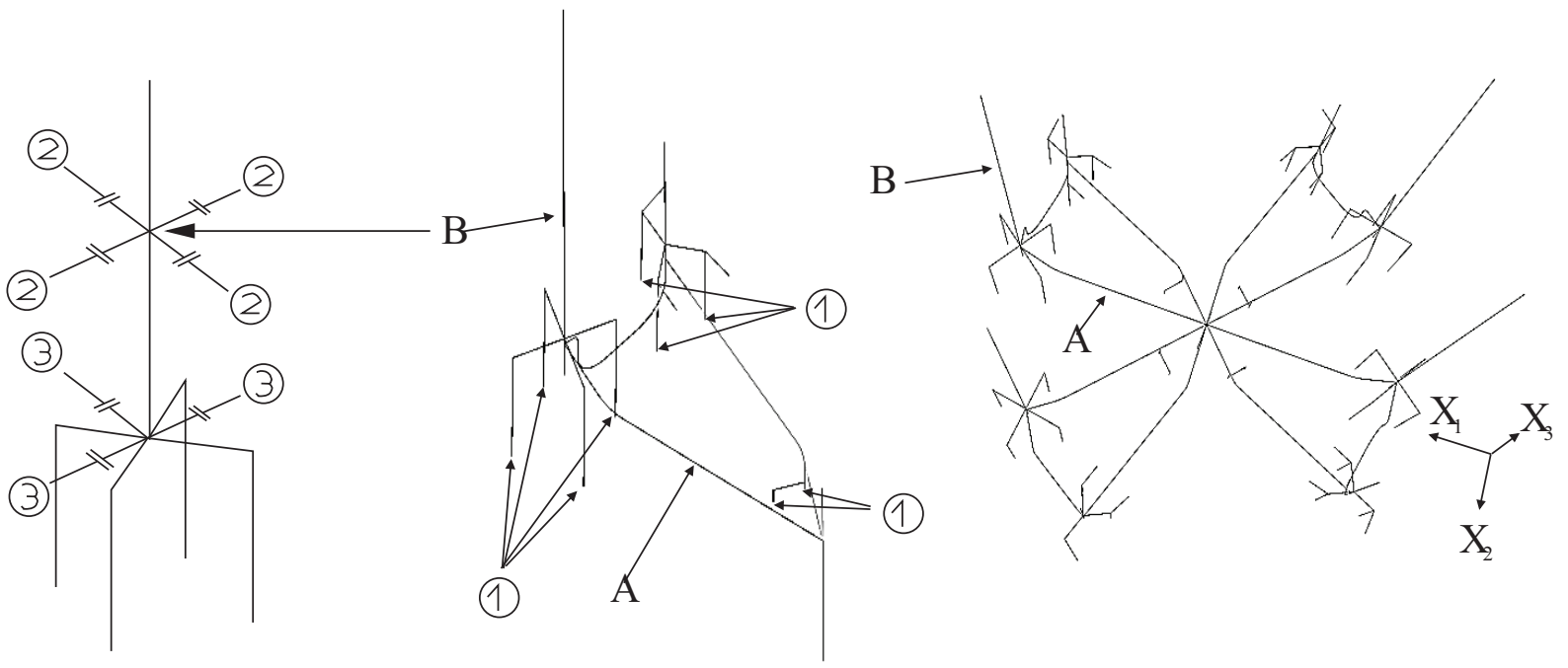

Figure 2. Finite element mesh of a steam generator (figure on the left), of one loop (figure on the middle) and of the four loops reactor coolant system (figure on the right). 


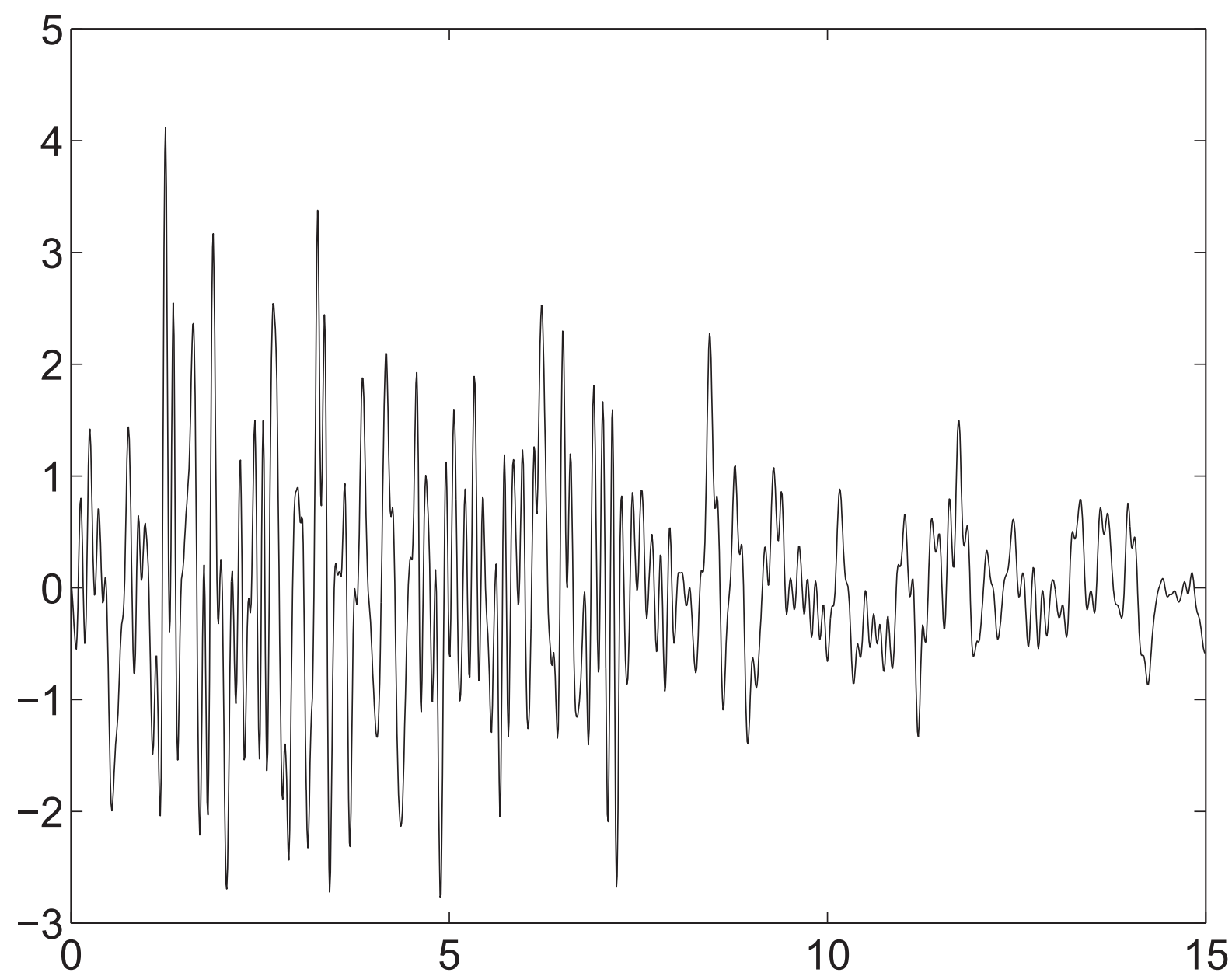

Figure 3. Example of accelerogram applied to one of the 36 supports of the four loops reactor coolant system (vertical axis in $\mathrm{m} / \mathrm{s}^{2}$, horizontal axis in seconds). 


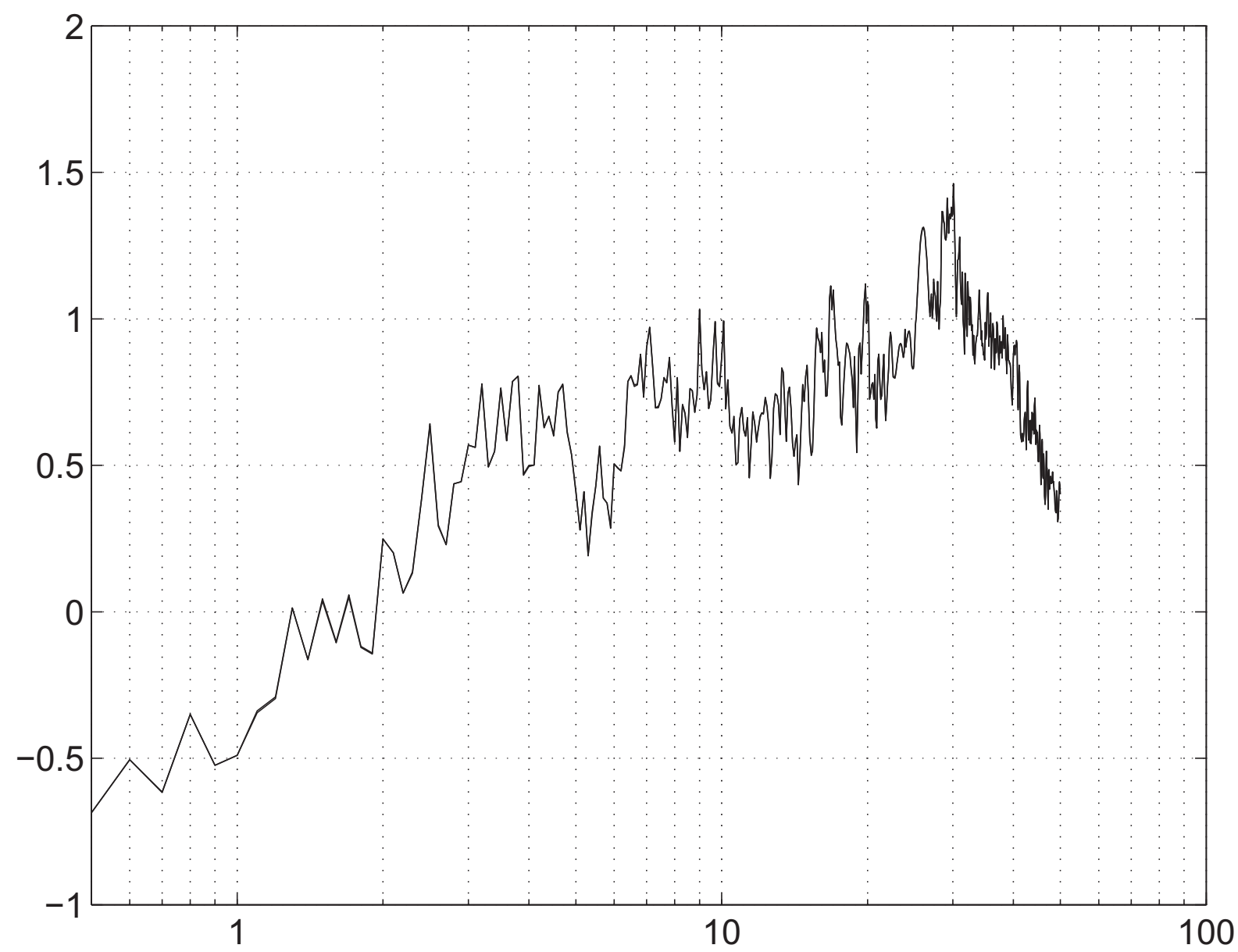

Figure 4. Normalized response spectrum in $\mathrm{dB}$ of the mean nonlinear dynamical system for DOF number $j_{s t o p}$ as a function of the frequency in $\mathrm{Hz}$ (horizontal axis). 


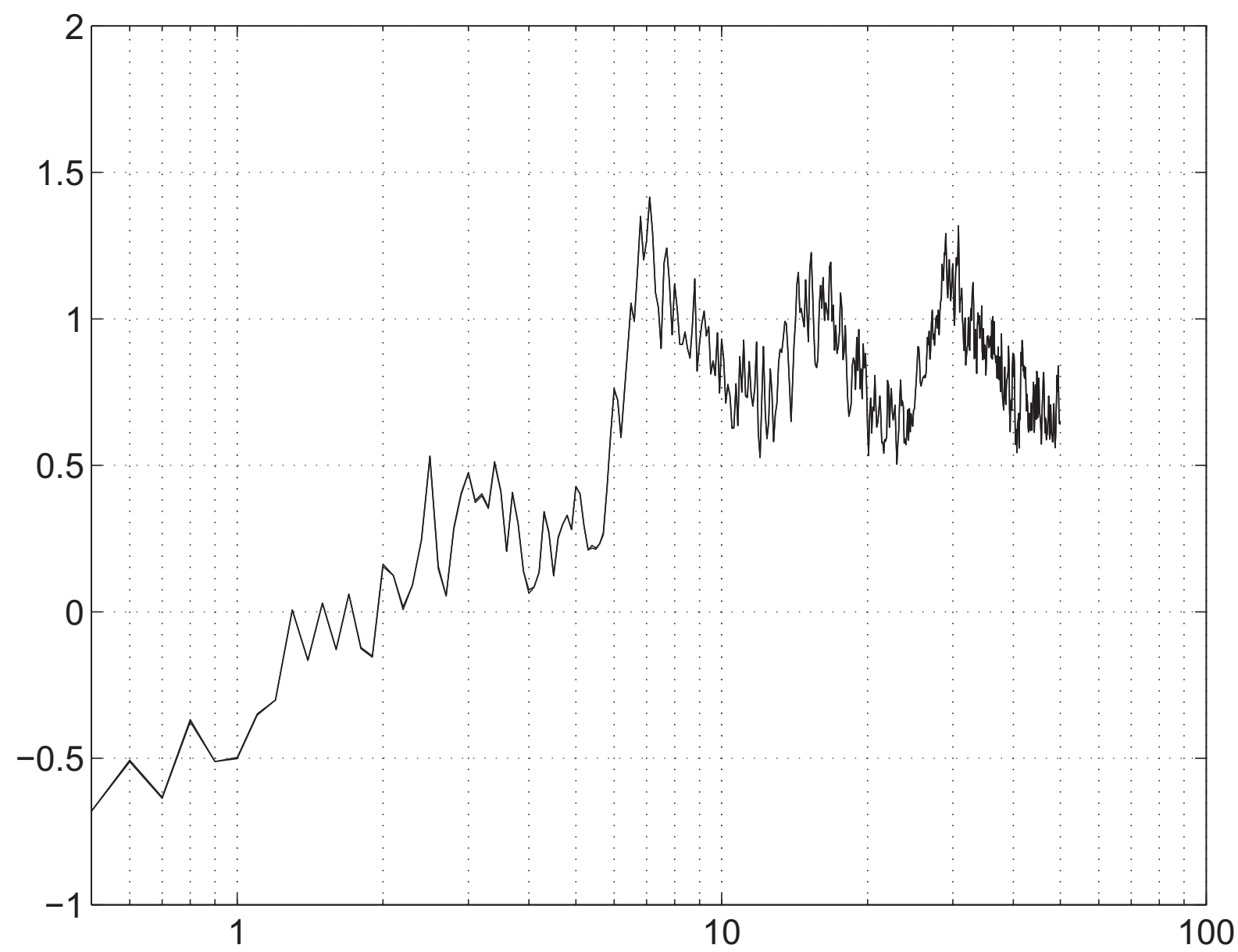

Figure 5. Normalized response spectrum in $\mathrm{dB}$ of the mean nonlinear dynamical system for DOF number $j_{o b s}$ as a function of the frequency in $\mathrm{Hz}$ (horizontal axis). 


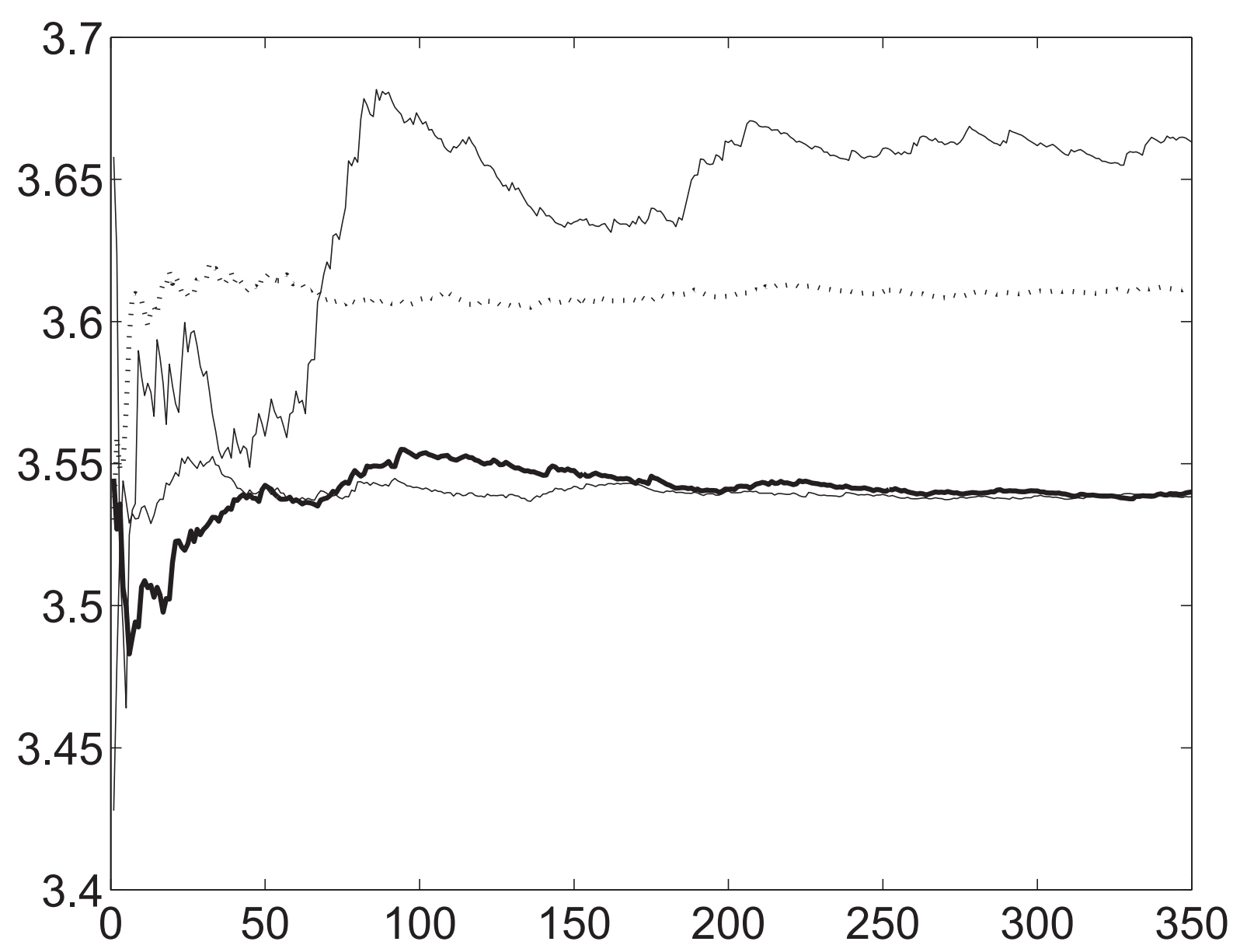

Figure 6. Graphs of functions $n_{s} \mapsto \log _{10}\left\{\operatorname{Conv}_{j_{\text {obs }}}\left(n_{s}, n\right)\right\}$ for $n=5$ (upper solid line), $n=50$ (thick dotted line), $n=100$ (lower thin solid line) and $n=200$ (lower thick solid line). 


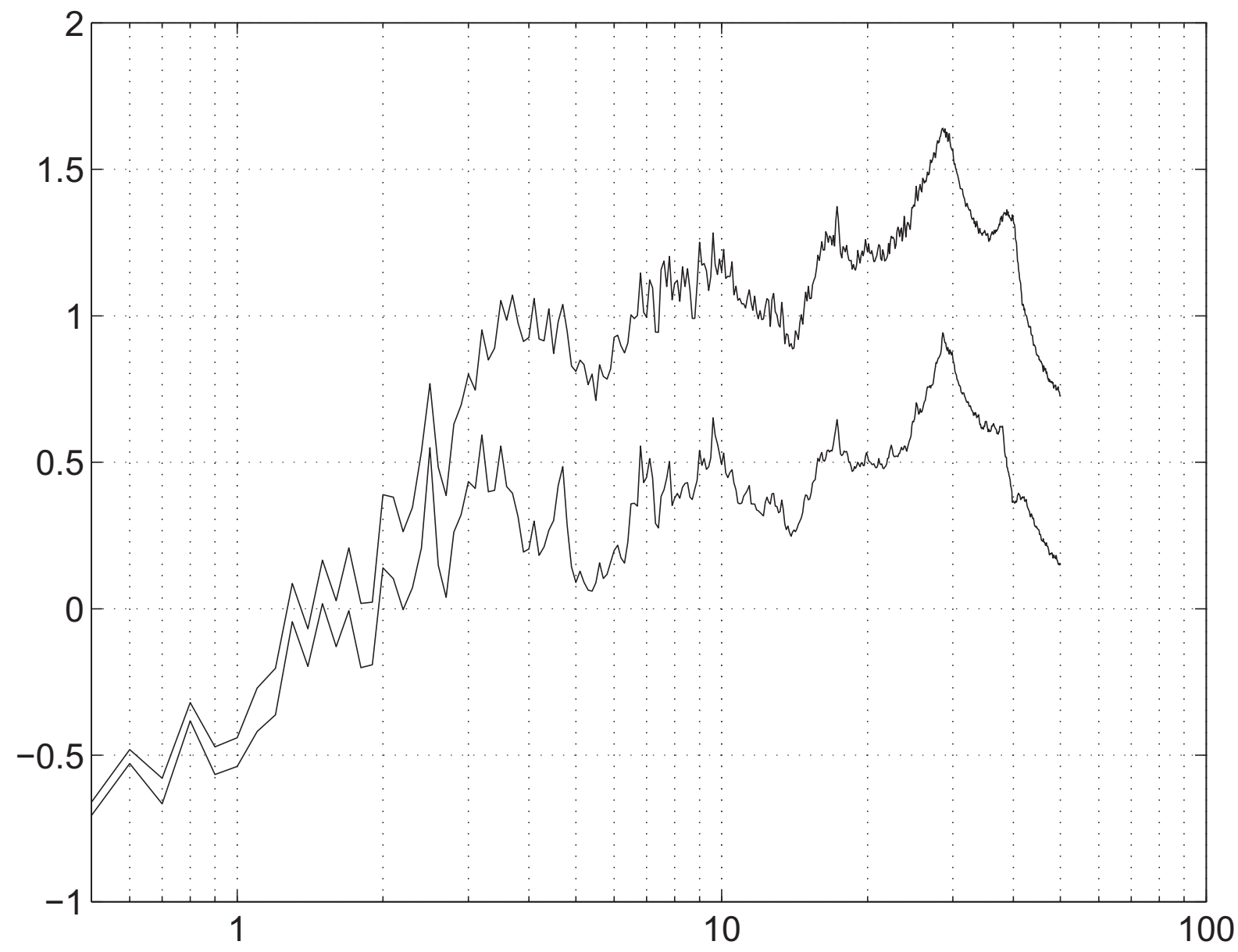

Figure 7. Confidence region for the normalized response spectrum in $\mathrm{dB}$ of DOF number $j_{\text {stop }}$ with a nonparametric and a parametric level of uncertainties equal to 0.2: upper envelope $d B_{j}^{+}(\xi, \nu)$ (upper solid line) and lower envelope $d B_{j}^{-}(\xi, \nu)$ (lower solid line) as a function of frequency $\nu$ in $\mathrm{Hz}$. 


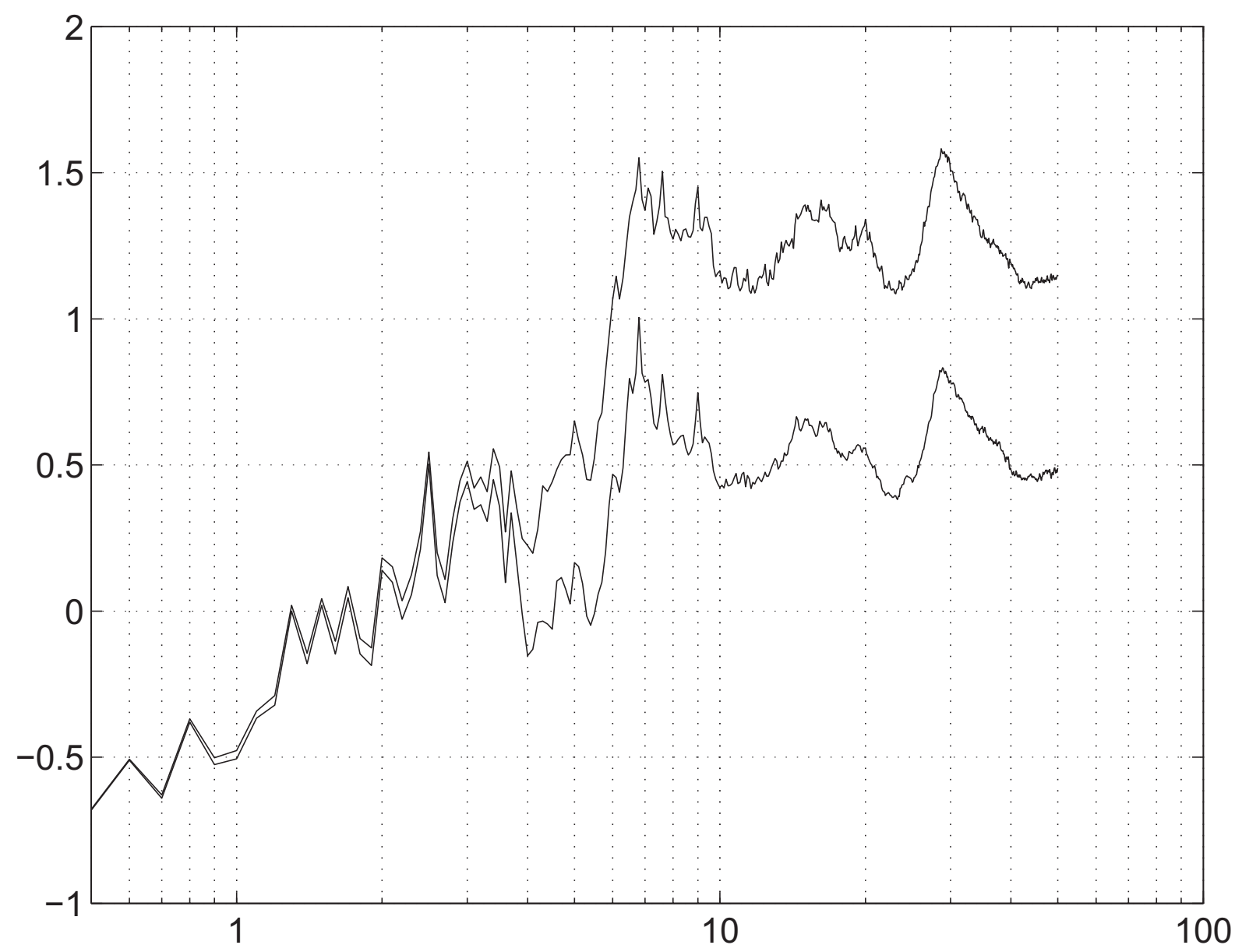

Figure 8. Confidence region for the normalized response spectrum in $\mathrm{dB}$ of DOF number $j_{o b s}$ with a nonparametric and a parametric level of uncertainties equal to 0.2. upper envelope $d B_{j}^{+}(\xi, \nu)$ (upper solid line) and lower envelope $d B_{j}^{-}(\xi, \nu)$ (lower solid line) as a function of frequency $\nu$ in $\mathrm{Hz}$. 


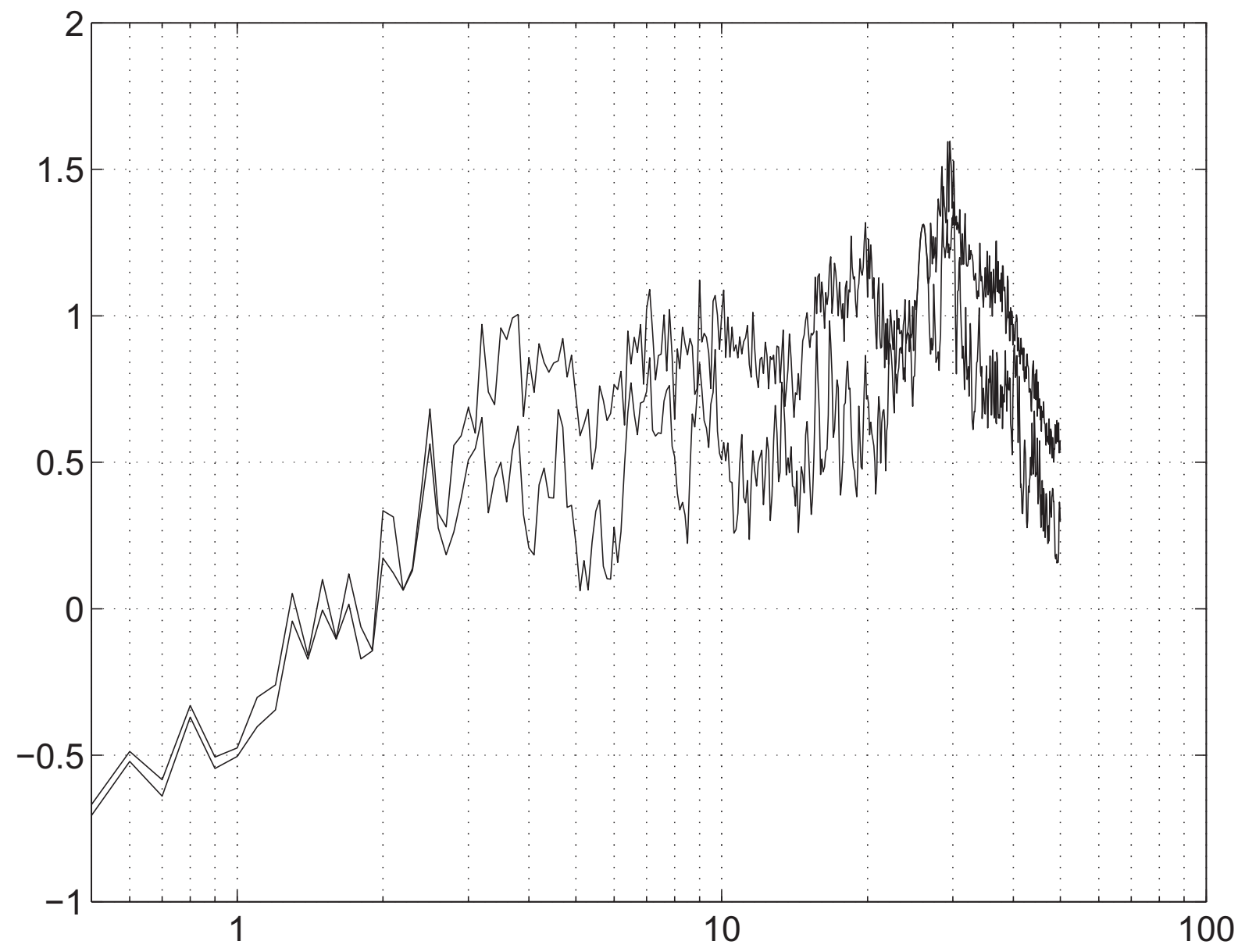

Figure 9. Confidence region for the normalized response spectrum in $\mathrm{dB}$ of DOF number $j_{\text {stop }}$ with a nonparametric equal to 0.00002 and a parametric level of uncertainties equal to 0 : upper envelope $d B_{j}^{+}(\xi, \nu)$ (upper solid line) and lower envelope $d B_{j}^{-}(\xi, \nu)$ (lower solid line) as a function of frequency $\nu$ in $\mathrm{Hz}$. 


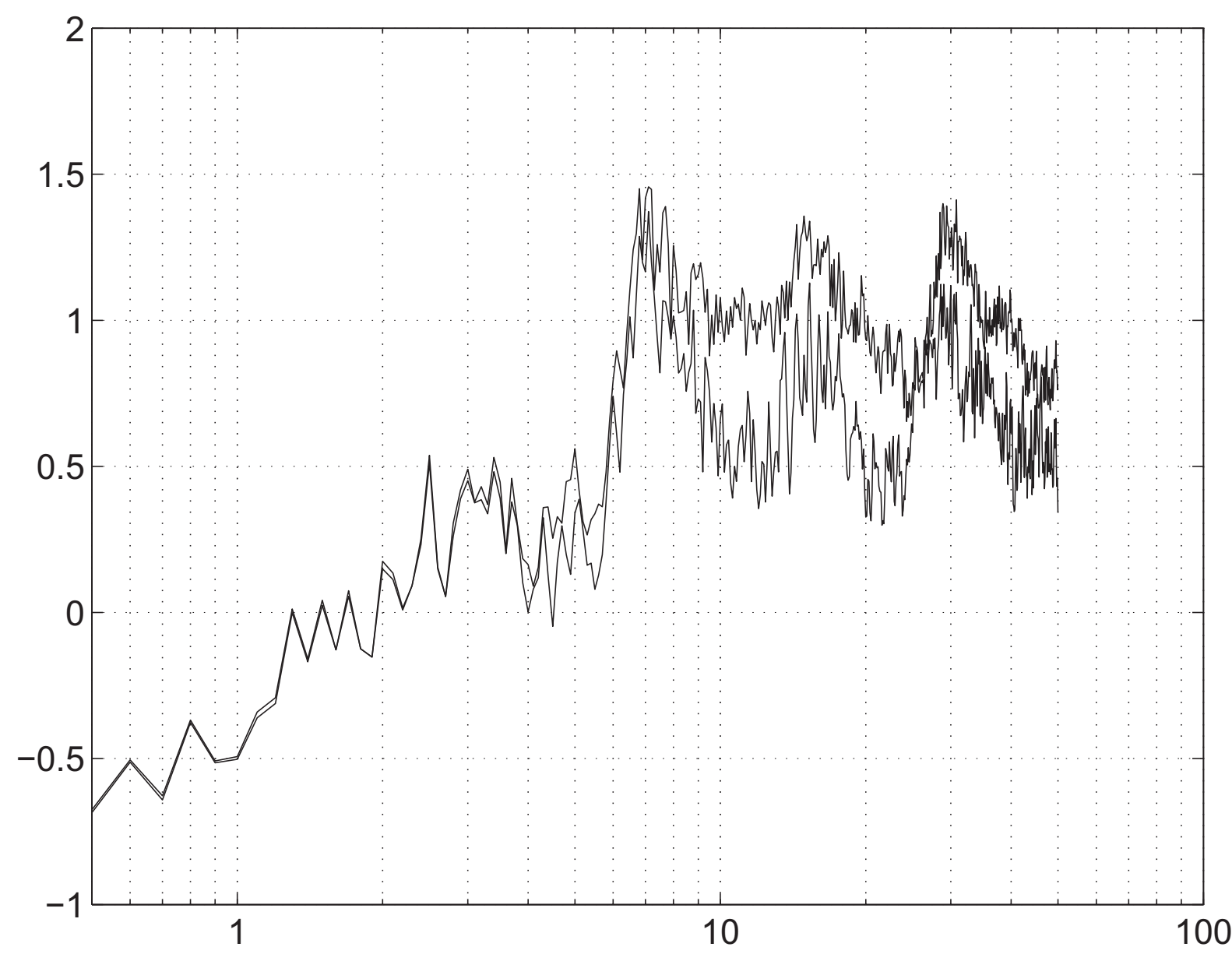

Figure 10. Confidence region for the normalized response spectrum in $\mathrm{dB}$ of DOF number $j_{\text {obs }}$ with a nonparametric equal to 0.00002 and a parametric level of uncertainties equal to 0: upper envelope $d B_{j}^{+}(\xi, \nu)$ (upper solid line) and lower envelope $d B_{j}^{-}(\xi, \nu)$ (lower solid line) as a function of frequency $\nu$ in $\mathrm{Hz}$. 